\title{
Eddy diffusion coefficients and their upper limits based on application of the similarity theory
}

\author{
M. N. Vlasov and M. C. Kelley \\ School of Electrical and Computer Engineering, Cornell University, Ithaca, New York, USA \\ Correspondence to: M. N. Vlasov (mv75@cornell.edu)
}

Received: 18 February 2015 - Accepted: 9 June 2015 - Published: 23 July 2015

\begin{abstract}
The equation for the diffusion velocity in the mesosphere and the lower thermosphere (MLT) includes the terms for molecular and eddy diffusion. These terms are very similar. For the first time, we show that, by using the similarity theory, the same formula can be obtained for the eddy diffusion coefficient as the commonly used formula derived by Weinstock (1981). The latter was obtained by taking, as a basis, the integral function for diffusion derived by Taylor (1921) and the three-dimensional Kolmogorov kinetic energy spectrum. The exact identity of both formulas means that the eddy diffusion and heat transport coefficients used in the equations, both for diffusion and thermal conductivity, must meet a criterion that restricts the outer eddy scale to being much less than the scale height of the atmosphere. This requirement is the same as the requirement that the free path of molecules must be much smaller than the scale height of the atmosphere. A further result of this criterion is that the eddy diffusion coefficients $K_{\text {ed }}$, inferred from measurements of energy dissipation rates, cannot exceed the maximum value of $3.2 \times 10^{6} \mathrm{~cm}^{2} \mathrm{~s}^{-1}$ for the maximum value of the energy dissipation rate of $2 \mathrm{~W} \mathrm{~kg}^{-1}$ measured in the mesosphere and the lower thermosphere (MLT). This means that eddy diffusion coefficients larger than the maximum value correspond to eddies with outer scales so large that it is impossible to use these coefficients in eddy diffusion and eddy heat transport equations. The application of this criterion to the different experimental data shows that some reported eddy diffusion coefficients do not meet this criterion. For example, the large values of these coefficients $\left(1 \times 10^{7} \mathrm{~cm}^{2} \mathrm{~s}^{-1}\right)$ estimated in the Turbulent Oxygen Mixing Experiment (TOMEX) do not correspond to this criterion. The $K_{\text {ed }}$ values inferred at high latitudes by Lübken (1997) meet this criterion for summer and winter polar data, but the $K_{\text {ed }}$ values for summer at low latitudes are larger than the
\end{abstract}

$K_{\text {ed }}$ maximum value corresponding to the criterion. Analysis of the experimental data on meteor train observations shows that energy dissipation with a small rate of about $0.2 \mathrm{~W} \mathrm{~kg}^{-1}$ sometimes can induce turbulence with eddy scales very close to the scale height of the atmosphere. Our results also explain the discrepancy between the large cooling rates calculated by Vlasov and Kelley (2014) and the temperatures given by the MSIS-E-90 model because, in these cases, the measured eddy diffusion coefficients used in calculating the cooling rates are larger than the maximum value presented above.

Keywords. Atmospheric composition and structure (middle atmosphere - composition and chemistry) - meteorology and atmospheric dynamics (middle atmosphere dynamics; turbulence)

\section{Introduction}

Problems exist in estimating the eddy diffusion and heat transport coefficients, $K_{\text {ed }}$ and $K_{\text {eh }}$, from experimental data. These problems are due to uncertainty in experimentally determining the turbulent energy dissipation rate and to the uncertainty of these coefficients' dependence on the energy dissipation rate $\varepsilon$, which is a key parameter in determining these coefficients from experimental data. Usually, the spectrum of density fluctuations inferred from experimental data and approximated using the theoretical model of Heisenberg (1948) facilitates determining the inner-scale $l_{0}$. This parameter is related to the Kolmogorov microscale, $\eta$, through the relation $l_{0}=9.9 \eta$ (Lübken, 1993). The Kolmogorov microscale is a rough estimate of the size of the smallest eddies that can provide turbulent energy dissipation with viscosity $v$. Then the $\varepsilon$ value can be calculated using the formula $\varepsilon=v^{3} \eta^{-4}$. Ac- 
cording to this formula, the $\varepsilon$ value strongly depends on the $\eta$ value, which is estimated by a rough approximation. For example, let us estimate the impact of $\eta$ values on the energy dissipation rate using the $l_{0}$ values inferred from the experimental data by Kelley et al. (2003). The $l_{0}$ values vary from 156 to $222 \mathrm{~m}$ and the $\varepsilon$ value can change from $0.14 \mathrm{~W} \mathrm{~kg}^{-1}$ to $0.58 \mathrm{~W} \mathrm{~kg}^{-1}$. Thus, the $40 \%$ increase in the $\eta$ value results in an $\varepsilon$ increase by a factor of 4.14 .

Additional uncertainty is caused by dependence of the eddy diffusion coefficient on the energy dissipation rate. The linear dependence $K_{\mathrm{ed}}=b \varepsilon / \omega_{\mathrm{B}}^{2}$ with $b=0.8$ and $\omega_{\mathrm{B}}$, the buoyancy frequency derived by Weinstock (1978), is commonly used to infer the $K_{\text {ed }}$ values from $\varepsilon$-measured values. However, the relation $b=R i /(P-R i)$, where $P$ and $R i$ are the Prandtl and Richardson numbers, respectively, can be obtained in the steady state using the stationary equation for the turbulent energy balance between the rate of energy transferred from the mean motion to the fluctuations on one side, and the rates of turbulent energy dissipation due to viscosity and the buoyancy force on the other side (Chandra, 1980; Gordiets et al., 1982). This balance assumes that the fluctuations are stationary, homogeneous, and isotropic. Weinstock's formula is also derived for the same conditions. However, Weinstock assumes that turbulence obtained in a region of dynamic instability $(R i \leq 0.25)$ will be transported by turbulent flux into regions of larger $R i$, and the $R i$ mean value may then be 0.44 , corresponding to $b=0.8$ for $P=1$. Note that if this transport is not possible, the $b$ value cannot exceed 0.3 . There is no evidence that either formula is better, but the latter has the problem of $R i$ determination.

In a previous paper (Vlasov and Kelley, 2014), we considered a set of eddy diffusion coefficients inferred from different experimental data. The difference between these eddy diffusion coefficients exceeded an order of magnitude. Also, a strong contradiction exists between the higher experimental coefficients and coefficients used in the typical modeling results (Hecht et al., 2004) because using large eddy diffusion coefficients generates unrealistic model results. Vlasov and Kelley (2014) showed that, by comparing the cooling rates calculated by the equation with the turbulent energy dissipation rate and eddy heat transport terms with cooling rates corresponding to temperatures given by the MSIS-E-90 model, it is possible to obtain the criterion for analyzing experimental data on the eddy heat transport coefficient. The coefficients that meet this criterion are found to be significantly less than a set of the coefficients inferred from experimental data using the well-known formula $K_{\text {ed }}=0.8 \varepsilon / \omega_{\mathrm{B}}^{2}$.

To our knowledge, published papers on estimating the eddy diffusion coefficient do not take into account the requirements corresponding to using this coefficient in diffusion and thermal conductivity equations. The diffusion equation includes the terms of molecular and eddy diffusion. These terms are very similar. In this paper, for the first time, the similarity theory is applied to infer the dependence of $K_{\text {ed }}$ on the energy dissipation rate and to determine the upper limit of the eddy diffusion coefficients. The latter is based on criteria for eddy scales corresponding to the diffusion equation usually used in models. The new criterion is applied to the analysis of some published experimental data.

\section{Application of the similarity theory for the eddy diffusion coefficient}

The commonly used equation for velocity induced by molecular and eddy diffusion in the upper atmosphere is given in the form of (Banks and Kockarts, 1973)

$$
\begin{aligned}
w= & -D_{\mathrm{m}}\left[\frac{1}{n_{i}} \frac{\partial n_{i}}{\partial z}+\frac{1}{H_{i}}+\left(1+\alpha_{T}\right) \frac{1}{T} \frac{\partial T}{\partial z}\right] \\
& -K_{\text {ed }}\left[\frac{1}{n_{i}} \frac{\partial n_{i}}{\partial z}+\frac{1}{H}+\frac{1}{T} \frac{\partial T}{\partial z}\right],
\end{aligned}
$$

where the molecular diffusion coefficient is given by the equation

$D_{\mathrm{m}}=(1 / 3) V_{\mathrm{th}}^{2} / v$

$V_{\mathrm{th}}^{2}=8 k_{\mathrm{b}} T /(\pi m)=8 E_{\mathrm{th}} / \pi$

where $E_{\mathrm{th}}=k_{\mathrm{b}} T m^{-1}$ is the thermal energy per unit of mass, $H$ and $H_{i}$ are the respective scale heights of the mixing gas and the $i$-th component of the gas, $V_{\text {th }}$ is the mean thermal velocity, $k_{\mathrm{b}}$ is the Boltzmann constant, $T$ is the temperature, $\alpha_{T}$ is dimensionless quantity for the thermal diffusion coefficient, $m$ is mass, and $v$ is the collision frequency. According to Eq. (1), $K_{\text {ed }}$ and $D_{\mathrm{m}}$ have the same dimension. According to the similarity theory, two physical phenomena, processes, or systems are similar if, at corresponding moments of time at corresponding points in space, the values of the variable quantities that characterize the state of one system are proportional to the corresponding quantities of the second system. The proportionality factor for each of the quantities is called the similarity factor. Following the similarity theory, the eddy diffusion coefficient $K_{\text {ed }}$ can be given by an equation similar to Eq. (2)

$K_{\text {ed }}=\xi_{1}(1 / 3) W_{\text {turb }}^{2} / \omega_{\mathrm{B}}$,

where $\xi_{1}$ is the similarity factor, $W_{\text {turb }}$ is the mean turbulent velocity, and $\omega_{\mathrm{B}}$ is the buoyancy frequency. Usually, the energy dissipation rate, $\varepsilon$, is measured in units of energy per unit of mass and per second. $\varepsilon=E_{\text {turb }} \omega_{\mathrm{B}}$ is used to determine the eddy diffusion coefficient and $W_{\text {turb }} E_{\text {turb }}$ can be considered the analog of $E_{\text {th }}$. Using the similarity of $V_{\text {th }}$ and $W_{\text {turb }}$ and Eq. (3), it is possible to obtain the equation

$W_{\text {turb }}^{2}=\xi_{2} \cdot 8 E_{\text {turb }} / \pi=\xi_{2} \cdot 8 \varepsilon /\left(\pi \cdot \omega_{\mathrm{B}}\right)$

and

$\varepsilon=\zeta_{2}(\pi / 8) W_{\text {turb }}^{2} \omega_{\mathrm{B}}$. 


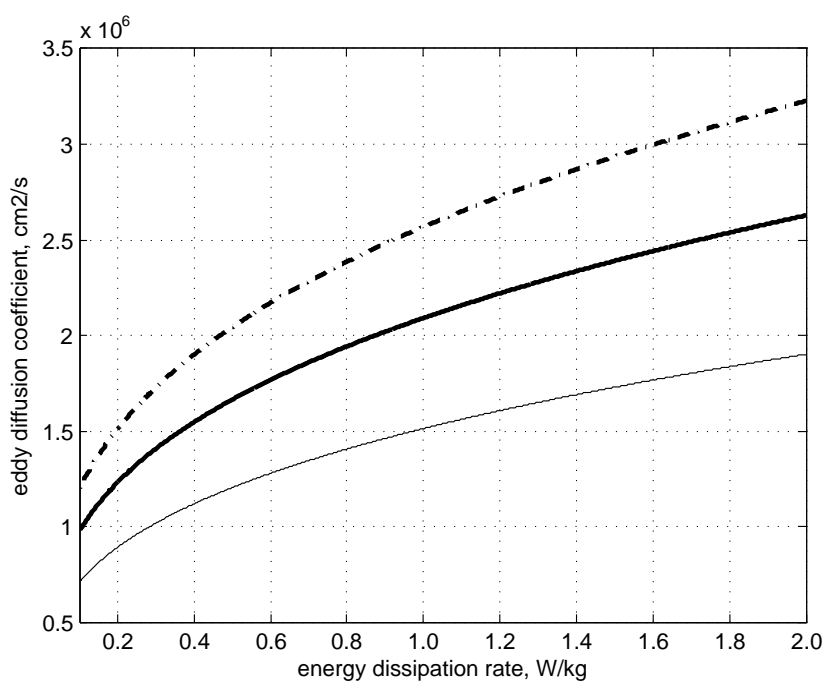

Figure 1. The $K_{\text {ed }}$ maximum value vs. the energy dissipation rate calculated by Eq. (12) with $b=0.8$ and $L_{B}=600$ and $700 \mathrm{~m}$ (thick solid and thick dashed-dotted curves, respectively), $b=0.3$ ( $P=1$, $R i=0.25$ ), and $L_{B}=600$ (thin solid curves).

Eq. (6) is the same as the equation

$\varepsilon=C_{0} \sigma_{w}^{2} \omega_{\mathrm{B}}$

obtained by Weinstock (1981), where $\sigma_{w}=W_{\text {turb }}$ is the mean turbulent velocity. This formula is derived by Weinstock (1981) using the three-dimensional Kolmogorov kinetic energy spectrum, which is valid when the eddy scales do not exceed the outer scale of turbulence.

$L_{B}=2 \pi \cdot W_{\text {turb }} / \omega_{\mathrm{B}}$

The main problem is in determining the dimensionless constant $C_{0}$ or $\xi_{2}(\pi / 8)$. Weinstock (1981) estimated $C_{0}=0.4-$ 5.0 and finally concluded that $C_{0}=0.4$ is more appropriate. In this case, $C_{0}$ is equal to $\xi_{2} \pi / 8=0.4$ for the similarity factor $\xi_{2}=1$. Therefore, there is excellent agreement between the formula derived by Weinstock and the formula derived using the approach based on the similarity theory. Substituting $W_{\text {turb }}$, given by Eq. (5), with $\xi_{2}=1$ into Eq. (4), it is possible to obtain

$K_{\text {ed }}=0.84 \varepsilon / \omega_{\mathrm{B}}^{2}$

for $\xi_{1}=1$. This equation is in excellent agreement with

$K_{\mathrm{eh}}=b \varepsilon / \omega_{\mathrm{B}}^{2}=0.8 \varepsilon / \omega_{\mathrm{B}}^{2}$

obtained by Weinstock (1978) and commonly used to estimate the coefficient of heat transport due to eddy turbulence $K_{\text {eh. }}$. For uniform turbulence, the coefficient $K_{\text {eh }}$ is equal to coefficient $K_{\text {ed }}$.

This result confirms application of the similarity theory. If we try to determine $K_{\text {ed }}$ corresponding to diffusion Eq. (1), the condition $L_{B}<<H$ must be met, and $L_{B} \leq 0.6 \mathrm{~km}$ because $H \leq 6 \mathrm{~km}$ in the upper mesosphere. This criterion is similar to criterion $\lambda<<H$ for molecular diffusion. Additionally, the smallest size of eddies $\eta$ must be much larger than the free path of molecules, and the $W_{\text {turb }}$ value must be much less than thermal velocity $V_{\text {th }} \approx 3.5 \times 10^{4} \mathrm{~cm} \mathrm{~s}^{-1}$. The $W_{\text {turb }}$ maximum value can be found to be $4.8 \times 10^{4} \mathrm{~cm} \mathrm{~s}^{-1}$ by using Eq. (8) for $L_{B}<H$ and the $\omega_{\mathrm{B}}$ maximum value of $5 \times 10^{-2} \mathrm{~s}^{-1}$. The latter value can be estimated using the relation $\omega_{\mathrm{B}}^{2}=R i S_{\max }^{2}$ for $R i \leq 0.25$ and the maximum wind shear, $S_{\max }=100 \mathrm{~m} \mathrm{~s}^{-1} \mathrm{~km}^{-1}$ (Larsen, 2002). Also, according to Eq. (8), the $W_{\text {turb }}$ value cannot exceed $4.8 \times 10^{4} \mathrm{~cm} \mathrm{~s}^{-1}$ for $L_{B}<<H$. Thus, the eddy diffusion velocity is much less than the thermal velocity. Note that our results show that the formula derived by Weinstock (1981) can be only applied for eddy diffusion; this formula cannot be used for turbulence with large-scale eddies.

According to experimental data (Lübken, 1997; Bishop et al., 2004; Szewczyk et al., 2013), the energy dissipation rate can range from 0.1 to $2 \mathrm{~W} \mathrm{~kg}^{-1}$. According to Eq. (8), the mean turbulent velocity can be given by the relation

$W_{\text {turb }}=L_{B} \cdot \omega_{\mathrm{B}} /(2 \pi)$

Substituting this relation into Eq. (4) and using Eq. (10) to determine $\omega_{\mathrm{B}}$, it is possible to obtain the equation

$K_{\mathrm{ed}}=\frac{L_{B}^{4 / 3}(b \varepsilon)^{1 / 3}}{2^{4 / 3}(8 \pi)^{2 / 3}}$.

As can be seen from the $K_{\text {eh }}$ value dependencies on the energy dissipation rate calculated by this formula and shown in Fig. 1, the $K_{\text {ed }}$ maximum value does not exceed $3.2 \times 10^{6} \mathrm{~cm}^{2} \mathrm{~s}^{-1}$ for the maximum value of the energy dissipation rate of $2 \mathrm{~W} \mathrm{~kg}^{-1}$ and $L_{B}=0.6 \mathrm{~km}$. This result is in good agreement with the $K_{\text {ed }}$ permissible limit corresponding to the criterion (Vlasov and Kelley, 2014) based on comparing the cooling rates produced by eddy turbulence with the normal cooling rates corresponding to the temperature given by the MSIS-E-90 model.

\section{The $K_{\text {ed }}$ upper limit and $K_{\text {ed }}$ values inferred from experimental data}

Using the Turbulent Oxygen Mixing Experiment (TOMEX) experimental data on the energy dissipation rate given in Table 1 and the $K_{\text {ed }}$ values given in Table 2 in Bishop et al. (2004), it is possible to estimate the $\omega_{\mathrm{B}}$ value corresponding to Eq. (10) used by Bishop et al. (2004). Combining Eq. (5) with $\xi_{2}=1$ and Eq. (8), the $L_{B}$ values can be calculated by the equation

$$
L_{B}=2 \sqrt{\frac{8 \pi b \varepsilon}{\omega_{\mathrm{B}}^{3}}} .
$$


(a)

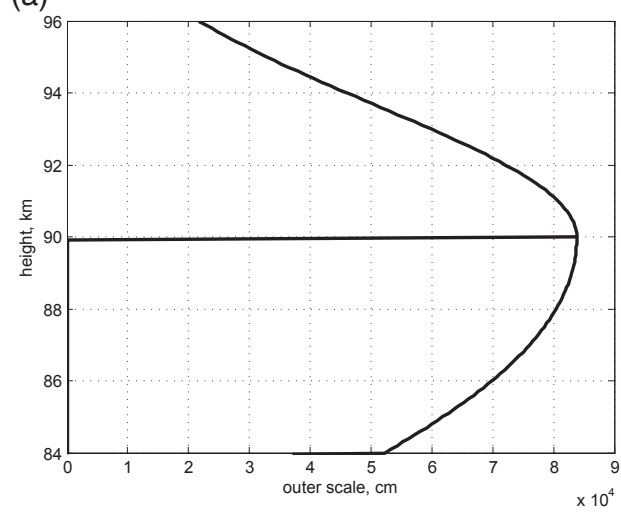

(b)

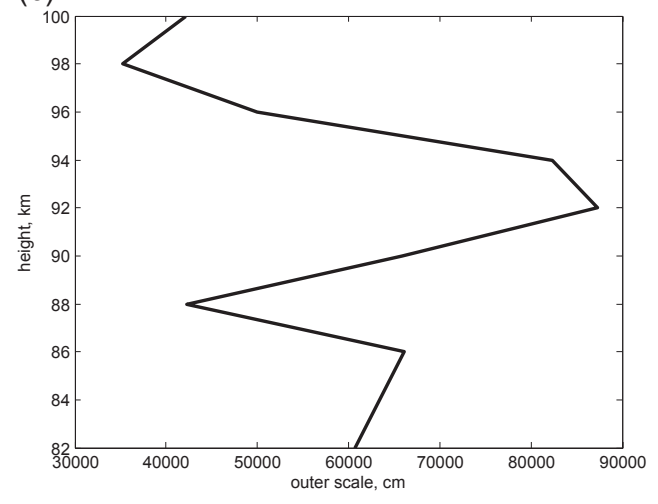

Figure 2. (a) The outer scale calculated by Eq. (14) with the $\varepsilon$ and $K_{\text {ed }}$ values given in Table 3 (summer) in Lübken (1997) and approximated, as can be seen from Figs. A1 and A2 in Appendix A. (b) The same as in Fig. 2a but using the $\varepsilon$ and $K_{\text {ed }}$ values in winter given in Table 4 in Lübken (1997).

The $L_{B}$ value can be found to be $3.8 \mathrm{~km}$ for $\varepsilon=0.41 \mathrm{~W} \mathrm{~kg}^{-1}$, as given in Table 1 in Bishop et al. (2004) at $102 \mathrm{~km}$ altitude. Using the temperature height profile measured during TOMEX at the time of Bishop's experiment as shown in Fig. 1 in Hecht et al. (2004), the $\omega_{\mathrm{B}}^{2}$ value can be found to be $1.52 \times 10^{-4} \mathrm{~s}^{-2}$ at $93 \mathrm{~km}$, and the $L_{B}$ value can be found to be $1.9 \mathrm{~km}$ for $\varepsilon=0.09 \mathrm{~W} \mathrm{~kg}^{-1}$, as given by Model 1 in Table 1 in Bishop et al. (2004). The $L_{B}$ value estimated for other models and altitudes in Table 1 can also be found to be larger than $1 \mathrm{~km}$. This means that the eddy diffusion coefficients inferred from these energy dissipation rates and given in Table 2 in Bishop et al. (2004) cannot be used in the diffusion equation because these coefficients correspond to turbulence with an outer eddy scale that is too large. Note that the TOMEX results are based on observation of the chemical tracer released by a rocket.

Rocket measurements of neutral density fluctuations were used by Lübken (1997) to infer the eddy diffusion coefficient. The $\varepsilon, W_{\text {turb }}$, and $K_{\text {ed }}$ mean values obtained in these experiments during summer are given in Table 3 in Lübken (1997). Using these parameters, Eqs. (10) and (5) with $\xi_{2}=1$, it is possible to estimate the $\omega_{\mathrm{B}}$ value. However, the $\omega_{\mathrm{B}}$ value corresponding to the $K_{\text {ed }}$ mean value at $90 \mathrm{~km}$ altitude is equal to $0.026 \mathrm{~s}^{-1}$, but the $\omega_{\mathrm{B}}$ value corresponding to the $W_{\text {turb }}$ mean value is equal to $0.047 \mathrm{~s}^{-1}$ at the same altitude. Perhaps this disagreement is a result of the averaging. The $L_{B}$ values calculated with the $W_{\text {turb }}$ mean value and $\omega_{\mathrm{B}}=0.047 \mathrm{~s}^{-1}$ can be found to be $382 \mathrm{~m}$. The $L_{B}$ height distribution calculated by the equation

$L_{B}=10.59 K_{\mathrm{ed}}^{3 / 4} / \varepsilon^{1 / 4}$,

obtained from Eq. (13) is shown in Fig. 2a. The approximations of the data on the $\varepsilon$ and $K_{\text {ed }}$ height distributions presented in Table 3 in Lübken (1997) are used in these calculations. A comparison of the approximations and data is shown in Figs. A1 and A2 in Appendix A. As can be seen

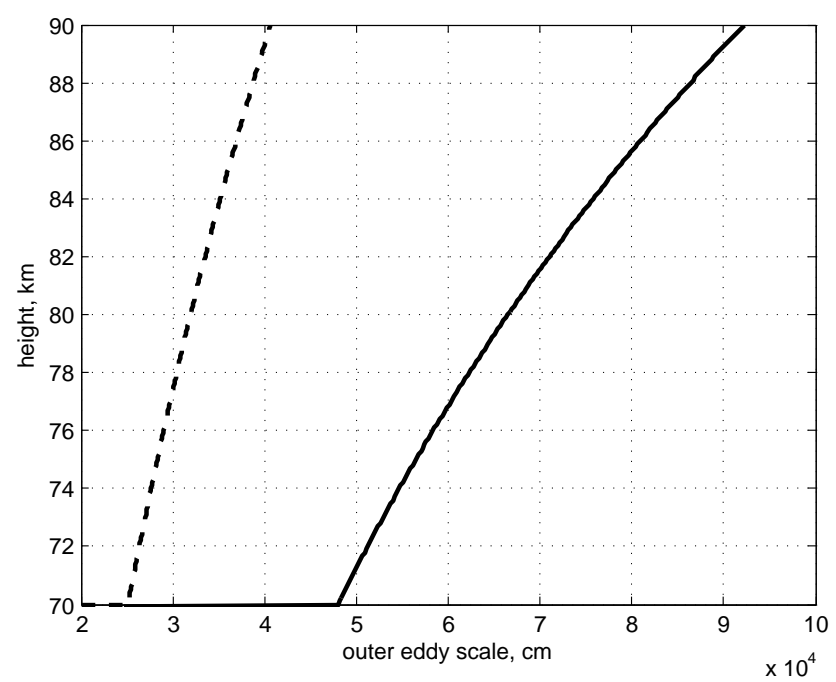

Figure 3. The height profiles of the outer scales of eddies $L_{B}$ in January (dashed curve) and September (solid curve) calculated using the data on $\varepsilon$ and $K_{\text {ed }}$ shown in Fig. 1 in Sasi and Vijayan (2001): $\varepsilon_{0}=31.6 \mathrm{erg} /(\mathrm{gs})$ and $K_{\mathrm{ed} 0}=1 \times 10^{5} \mathrm{~cm}^{2} \mathrm{~s}^{-1}$ for January and $\varepsilon_{0}=75 \mathrm{erg} /(\mathrm{gs})$ and $K_{\mathrm{ed} 0}=3.16 \times 10^{5} \mathrm{~cm}^{2} \mathrm{~s}^{-1}$ for September.

from the $L_{B}$ height profiles shown in Fig. 2a and b, there is no significant difference between the outer scales calculated with Lübken's summer and winter data. In general, the $K_{\text {ed }}$ inferred by Lübken (1997) meets the criterion for the $K_{\text {ed }}$ maximum value given in Eq. (12) and shown in Fig. 1 for use in the diffusion equation.

Data on the turbulent energy dissipation rates were obtained by the Indian mesosphere-stratosphere-troposphere (MST) radar located at Gadanki $\left(13.5^{\circ} \mathrm{N}, 79.2^{\circ}\right.$ E) during a 3 -year period. The eddy diffusion coefficients have been estimated using Eq. (10) (Sasi and Vijayan, 2001). The height distributions of the $\varepsilon$ and $K_{\text {ed }}$ mean values for the differ- 


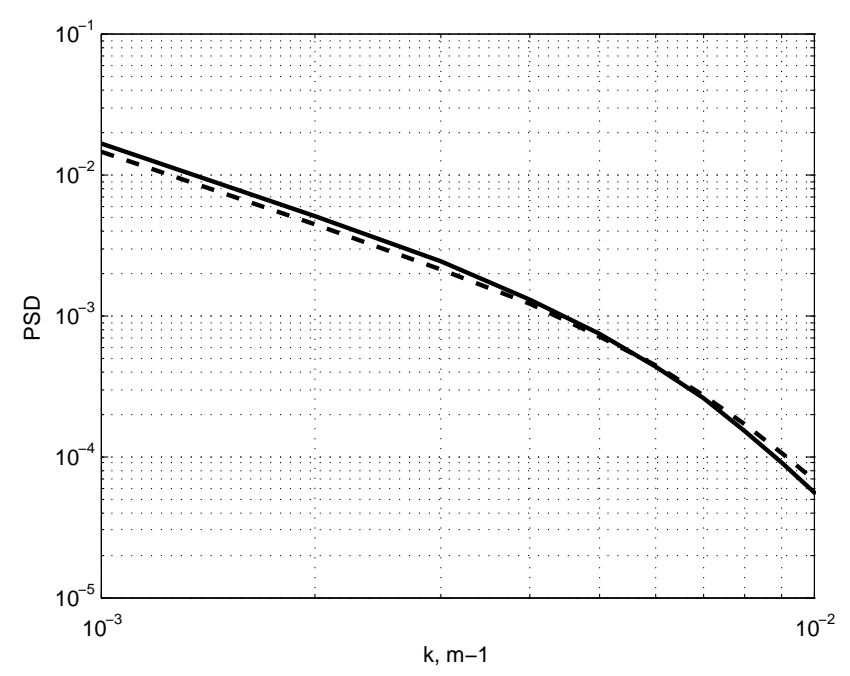

Figure 4. The power spectra of the relative $\mathrm{Na}$ density fluctuations calculated by Eq. (18) with $N=5 \times 10^{-7} \mathrm{~s}^{-1}, A=0.216, v=16.6$ $\mathrm{m}^{2} \mathrm{~s}^{-1}$, and $k_{0}=0.0084 \mathrm{~m}^{-1}$ and $0.0094 \mathrm{~m}^{-1}$ (solid and dashed curves, respectively).

ent months were approximated by exponential functions, as can be seen from Fig. 1 in Sasi and Vijayan (2001). Using these distributions and Eq. (14), it is possible to calculate the height distributions of the $L_{B}$ values shown in Fig. 3. These results show that the criterion $L_{B}<<H$ is met at all altitudes in winter but this criterion is only met at altitudes above $80 \mathrm{~km}$ in summer at lower latitudes. Note that $L_{B}$ values at high latitude calculated with the $\varepsilon$ and $K_{\text {ed }}$ values given by Lübken (1997) do not show significant seasonal variations.

Kelley et al. (2003, hereafter referred to as K03) presented additional experimental data. The time evolution of persistent meteor trains was used to determine the eddy diffusion coefficient in the upper mesosphere. The sodium density in the train was sufficient to use it as a passive scalar tracer of turbulence. The simultaneous measurements of the power spectrum of relative $\mathrm{Na}$ density fluctuations, the neutral temperature, and wind are presented within the altitude range of $83.5-100 \mathrm{~km}$. Also, they estimated the $\omega_{\mathrm{B}}^{2}$ and $R i$ values corresponding to the measured temperature and wind. In our analysis, we use the averaged spectra shown in Fig. 7 in K03. This spectrum can be approximated by the equation

$P(k)=\frac{\Gamma(5 / 3) \sin (\pi / 3) \alpha^{2} N}{2 \pi v\left(9.9 k_{0}\right)^{4 / 3}} \times \frac{k^{-5 / 3}}{\left[1+\left(k / k_{0}\right)^{8 / 3}\right]^{2}}$,

obtained by substituting the relation

$\varepsilon=v^{3}\left(9.9 k_{0}\right)^{4}$

into the theoretical turbulent spectrum presented by Heisenberg (1948):

$P(k)=\frac{\Gamma(5 / 3) \sin (\pi / 3) \alpha^{2} N}{2 \pi \varepsilon^{1 / 3}} \times \frac{k^{-5 / 3}}{\left[1+\left(k / k_{0}\right)^{8 / 3}\right]^{2}}$, where $v$ is the kinematic viscosity, $\frac{\Gamma(5 / 3) \sin (\pi / 3) \alpha^{2}}{2 \pi}=$ $0.216=A$ is the constant coefficient (see Lübken, 1993), $k_{0}$ is the wave number corresponding to the inner scale of eddies $l_{0}^{H}$, and $N$ represents the amount of inhomogeneity that disappears per unit time due to molecular diffusion (Lübken, 1997). These latter two parameters can be used as the fit coefficients. The averaged altitude corresponding to the averaged spectrum can be found to be $98 \mathrm{~km}$, according to the height profiles of the Na density shown in Fig. 6 in K03. The kinematic viscosity can be found to be $1.66 \times 10^{5} \mathrm{~cm}^{2} \mathrm{~s}^{-1}$ using the formulas $v=\mu / \rho$ and $\mu=3.43 \times 10^{-6} T^{0.69} \mathrm{~g}(\mathrm{~cm} \times \mathrm{s})$ (Banks and Kockarts, 1973), and $\rho=8.1 \times 10^{-10} \mathrm{~g} \mathrm{~cm}^{-3}$ is the density at $98 \mathrm{~km}$, according to the MSIS-E- 90 model.

The power spectra calculated by Eq. (15) are shown in Fig. 4. The spectrum calculated with $k_{0}=0.0084 \mathrm{~m}^{-1}$ and $N=5 \times 10^{-7} \mathrm{~s}^{-1}$ provides the best fit to the averaged experimental spectrum shown in Fig. 7 in K03. In this case, the energy dissipation rate can be found to be $0.22 \mathrm{~W} \mathrm{~kg}^{-1}$ according to Eq. (16). The eddy diffusion coefficient calculated by Eq. (10) with $\omega_{B}^{2}=1 \times 10^{-4} \mathrm{~s}^{-2}$ given at $98 \mathrm{~km}$ in Fig. $8 \mathrm{c}$ in $\mathrm{K} 03 \mathrm{can}$ be found to be $1.76 \times 10^{7} \mathrm{~cm}^{2} \mathrm{~s}^{-1}$. However, using the temperature height profile given in Fig. 8a, the $\omega_{\mathrm{B}}^{2}$ value can be found to be $1.7 \times 10^{-4} \mathrm{~s}^{-2}(T=170 \mathrm{~K}$, and $\partial T / \partial z=6.6 \mathrm{~K} \mathrm{~km}^{-1}$ ) and $K_{\text {ed }}=1.04 \times 10^{7} \mathrm{~cm}^{2} \mathrm{~s}^{-1}$ in this case. However, this coefficient calculated by Eq. (10) with $b=R i / P-R i)$ instead of $b=0.8$ with $P=1$ and $R i=0.2$ given in Fig. $8 \mathrm{~d}$ in $\mathrm{K} 03$ can be found to be $3.2 \times 10^{6} \mathrm{~cm}^{2} \mathrm{~s}^{-1}$. This coefficient is larger than the maximum value corresponding to Fig. 1 for $\varepsilon=0.22 \mathrm{~W} \mathrm{~kg}^{-1}$, and the $L_{B}$ value calculated by Eq. (13) for $\varepsilon=0.22 \mathrm{~W} \mathrm{~kg}^{-1}$ and $\omega_{\mathrm{B}}^{2}=$ $1.7 \times 10^{-4} \mathrm{~s}^{-2}$ is equaled to $1.6 \mathrm{~km}$. This value is comparable with the atmospheric-scale height, which means that eddies with large scales must occur and that the eddy diffusion coefficient inferred from these experimental data cannot be used in the diffusion and heat conductivity equations. Note in this case that turbulent fluctuations may be inhomogeneous, non-isotropic, and stationary. This can be seen from the color figures presented in K03. Recently, Kelley et al. (2009) suggested that the MLT is characterized by two-dimensional turbulence, which is in agreement with the current results.

\section{Conclusions}

For the first time, using similarity theory, the formulas for the eddy diffusion coefficient and the turbulent energy dissipation rate have been obtained, and these formulas coincide with the commonly used formulas derived by Weinstock $(1978,1981)$. The latter formula was derived using the integral function for diffusion derived by Taylor (1921) and the three-dimensional Kolmogorov kinetic energy spectrum. This result means that the eddy diffusion and heat transport coefficients used in the equations for diffusion and thermal conductivity must meet all of the following criteria: 
1. the minimum eddy scale is much larger than the mean free path of molecules,

2. the outer eddy scale is much less than the scale height of atmospheric gas,

3. the mean turbulent velocity $W_{\text {turb }}$ must be much less than the thermal velocity.

Using criterion (2) and the dependencies of the eddy diffusion coefficient on energy dissipation rate $\varepsilon$ and the outerscale $L_{B}$, it is shown that $K_{\text {ed }}$ maximum values cannot exceed $3.2 \times 10^{6} \mathrm{~cm}^{2} \mathrm{~s}^{-1}$ for the $\varepsilon$ maximum value of $2 \mathrm{~W} \mathrm{~kg}^{-1}$. The dependence of the $K_{\text {ed }}$ maximum value on the energy dissipation rate is shown in Fig. 1. This result means that the eddy heat transport and eddy diffusion coefficients inferred from measurements of the energy dissipation rates, which exceed the maximum value, cannot be used in the thermal balance and diffusion equations because the eddies have unreasonably large vertical scales. These $K_{\text {ed }}$ maximum values are in excellent agreement with the values estimated by Vlasov and Kelley (2014) using the cooling rates induced by eddy heat transport. For example, the $K_{\text {ed }}$ large values of $(5 \div 20) \times 10^{6} \mathrm{~cm}^{2} \mathrm{~s}^{-1}$ inferred by Bishop et al. (2004) from observations of chemical tracers released by a rocket during the TOMEX do not meet criterion Eq. (2) because of the very large eddy scales $\left(L_{B}>3 \mathrm{~km}\right)$. At the same time, the cooling rates calculated with these coefficients are much higher than normal cooling rates corresponding to the temperature given by the MSIS-E- 90 model. This result was shown by Vlasov and Kelley (2014).
Analysis of the experimental data (Lübken, 1997) shows that the eddy diffusion coefficients inferred from rocket measurements of density fluctuations at high latitudes correspond to the criteria described above and that the $L_{B}$ values do not show significant seasonal variations in the polar region. However, a very significant difference between the $L_{B}$ summer and winter values estimated from the MST radar data (Sasi and Vijayan, 2001) was found at low latitudes.

Our analysis of the Kelley at al. (2003) results, based on observations of the time evolution of persistent meteor trains (Kelley at al., 2003), shows that eddies with very large scales may occur despite the fact that a very small energy dissipation rate was found. Due to the large scales, the eddy diffusion coefficient inferred from these data does not meet the criterion corresponding to the diffusion equation. Also, our results support the notion that two-dimensional turbulence is a characteristic of the MLT (Kelley et al., 2009).

The topical editor A. J. Kavanagh thanks C. Gardner and one anonymous referee for help in evaluating this paper. 


\section{Appendix A}

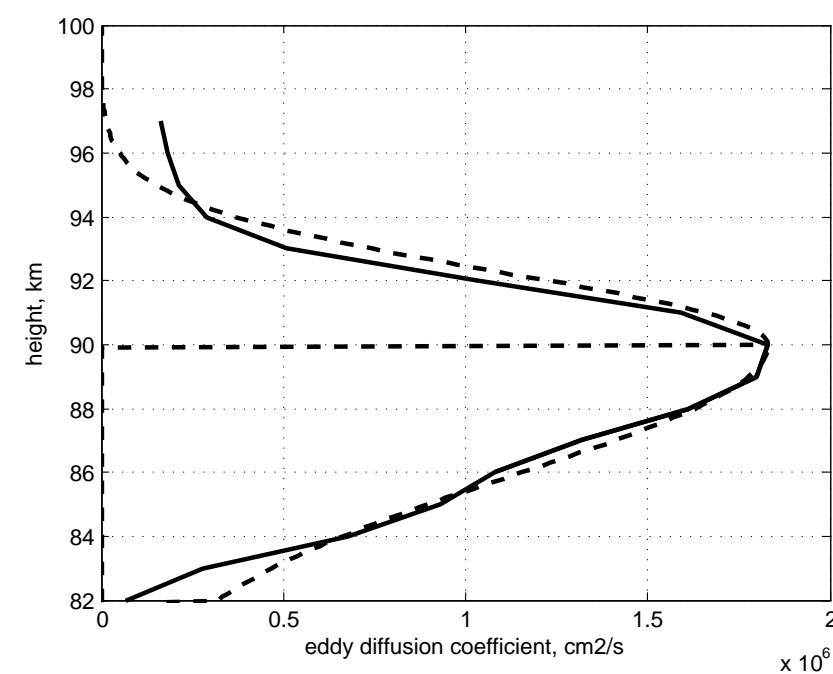

Figure A1. The eddy diffusion coefficient inferred by Lübken (1997) in polar summer (solid curve) and approximated by Eqs. (A1) and (A2) (dashed curve).

The eddy diffusion coefficient inferred by Lübken (1997) from measurements of the turbulent energy dissipation rate in the summer polar mesosphere can be approximated by formulas suggested by Shimazaki (1971):

$$
\begin{aligned}
K_{\mathrm{ed}} & =K_{\mathrm{ed}}^{0} \exp \left[S_{1}\left(z-z_{\mathrm{m}}\right)\right] \\
& +\left(K_{\mathrm{ed}}^{\mathrm{m}}-K_{\mathrm{ed}}^{0}\right) \exp \left[-S_{2}\left(z-z_{\mathrm{m}}\right)^{2}\right] \quad \mathrm{z} \leq \mathrm{z}_{\mathrm{m}},
\end{aligned}
$$

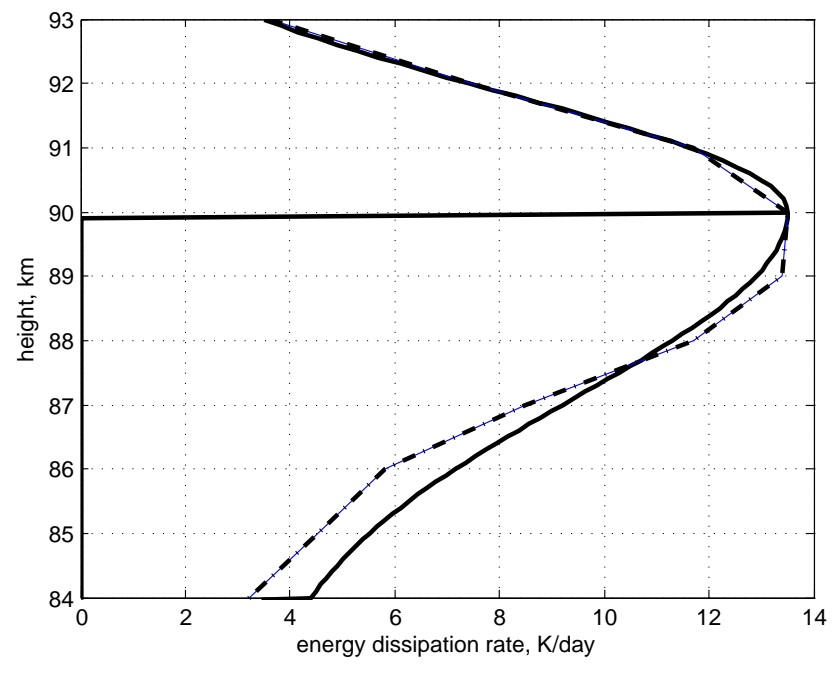

Figure A2. The height profiles of the energy dissipation rate measured by Lübken (1997) (dashed curve) and approximated by Eqs. (A1) and (A2) suggested by Shimazaki (1971) (solid curve) with $\varepsilon_{\mathrm{m}}=13.5 \mathrm{~K} \mathrm{day}^{-1}$ instead of $K_{\mathrm{ed}}^{\mathrm{m}}$ and $S_{1}=0.01 \mathrm{~km}^{-1}$, $S_{2}=0.06 \mathrm{~km}^{-2}$, and $S_{3}=0.1 \mathrm{~km}^{2}$.

$K_{\mathrm{ed}}=K_{\mathrm{ed}}^{\mathrm{m}} \exp \left[-S_{3}\left(z-z_{\mathrm{m}}\right)^{2}\right] \quad \mathrm{z}>\mathrm{z}_{\mathrm{m}}$,

where $K_{\mathrm{ed}}^{\mathrm{m}}=1.83 \times 10^{6} \mathrm{~cm}^{2} \mathrm{~s}^{-1}$ is the maximum of these coefficients, $z_{\mathrm{m}}=90 \mathrm{~km}, S_{1}=0.05 \mathrm{~km}^{-1}, S_{2}=0.03 \mathrm{~km}^{-2}$, and $S_{3}=0.1 \mathrm{~km}^{-2}$ (see Fig. A1). This approximation can also be used for the energy dissipation rate (see Fig. A2). 


\section{References}

Banks, P. M. and Kockarts, G.: Aeronomy, Academic Press, New York, USA, 1973.

Bishop, R. L., Larsen, M. F., Hecht, J. H., Liu, A. Z., and Gardner, C. S.: TOMEX: Mesospheric and lower thermospheric diffusivity and instability layers, J. Geophys. Res., 109, D02S03, doi:10.1029/2002JD003079, 2004.

Chandra, S.: Energetics and thermal structure of the middle atmosphere, Planet. Space Sci., 28, 585-593, 1980.

Gordiets, B. F., Kulikov, Y. N., Markov, M. N., and Marov, M. Y.: Numerical modeling of the thermospheric heat budget, J. Geophys. Res., 87, 4504-4514, 1982.

Hecht, J. H., Liu, A. Z., Walterscheid, R. L., Roble, R. G., Larsen, M. F., and Clemmons, J. H.: Airglow emissions and oxygen mixing ratios from the photometer experiment on the Turbulent Oxygen Mixing Experiment (TOMEX), J. Geophys. Res., 109, D02S05, 2004.

Heisenberg, W.: Zur statistischen Theorie der Turbulenz, Z. Phys., 124, 628-657, 1948.

Kelley, M. C., Kruschwitz, C. A., Gardner, C. S., Drummond, J. D., and Kane, T. J.: Mesospheric turbulence measurements from persistent Leonid meteor train observations, J. Geophys. Res., 108, 8454, doi:10.1029/2002JD002392, 2003.

Kelley, M. C., Seyler, C. E., and Larsen, M. F.: Two-dimensional turbulence, space shuttle plume transport in the thermosphere, and a possible relation to the Great Siberian Impact Event, Geophys. Res. Lett., 36, L14103, doi:10.1029/2009GL038362, 2009.

Larsen, M. F.: Winds and shears in the mesosphere and lower thermosphere: Results from four decades of chemical release wind measurements, J. Geophys. Res., 107, 1215, doi:1029/2001JA000218, 2002.
Lübken, F. J.: Seasonal variation of turbulent energy dissipation rates at high latitudes as determined by in situ measurements of neutral density fluctuations, J. Geophys. Res., 102, 1344113456, 1997.

Lübken, F. J., Hillert, W., Lehmacher, G., and von Zahn, U.: Experiments revealing small impact of turbulence on the energy budget of the mesosphere and lower thermosphere, J. Geophys. Res., 98, 20369-20384, 1993.

Sasi, M. N. and Vijayan, L.: Turbulence characteristics in the tropical mesosphere as obtained by MST radar at Gadanki $\left(13.5^{\circ} \mathrm{N}\right.$, 79. ${ }^{\circ} \mathrm{E}$ ), Ann. Geophys., 19, 1019-1025, doi:10.5194/angeo-191019-2001, 2001.

Shimazaki, T.: Effective eddy diffusion coefficient and atmospheric composition in the lower thermosphere, J. Atmos. Terr. Phys., 33, 1383-1401, 1971.

Szewczyk, A., Strelnikov, B., Rapp, M., Strelnikova, I., Baumgarten, G., Kaifler, N., Dunker, T., and Hoppe, U.-P.: Simultaneous observations of a Mesospheric Inversion Layer and turbulence during the ECOMA-2010 rocket campaign, Ann. Geophys., 31, 775-785, 2013, http://www.ann-geophys.net/31/775/2013/.

Taylor, G. I.: Diffusion by continuous movements, Proc. London Math. Soc., 20, 196-212, 1921.

Vlasov, M. N. and Kelley, M. C.: Criterion for analyzing experimental data on eddy diffusion coefficients, Ann. Geophys., 32, 581-588, doi:10.5194/angeo-32-581-2014, 2014.

Weinstock, J.: Vertical turbulent diffusion in a stably stratified fluid, J. Atmos. Sci., 35, 1022-1027, 1978.

Weinstock, J.: Energy dissipation rates of turbulence in the stable free atmosphere, J. Atmos. Sci., 38, 880-883, 1981. 